\title{
Additional chemotherapy improves survival in stage II-III pulmonary sarcomatoid carcinoma patients undergoing surgery: a propensity scoring matching analysis
}

\author{
Yanhong Cen ${ }^{1 \# \wedge}$, Chunxu Yang ${ }^{1 \#}$, Jiangbo Ren $^{2}$, Yan Gong ${ }^{2} \wedge$, Conghua Xie ${ }^{1,3,4} \wedge$ \\ ${ }^{1}$ Department of Radiation and Medical Oncology, Zhongnan Hospital of Wuhan University, Wuhan, China; ${ }^{2}$ Department of Biological Repositories, \\ Zhongnan Hospital of Wuhan University, Wuhan, China; ${ }^{3}$ Hubei Key Laboratory of Tumor Biological Behaviors, Zhongnan Hospital of Wuhan \\ University, Wuhan, China; ${ }^{4}$ Hubei Cancer Clinical Study Center, Zhongnan Hospital of Wuhan University, Wuhan, China \\ Contributions: (I) Conception and design: Y Cen, Y Gong, C Xie; (II) Administrative support: Y Gong, C Xie; (III) Provision of study materials \\ or patients: Y Cen, C Yang; (IV) Collection and assembly of data: Y Cen, J Ren; (V) Data analysis and interpretation: Y Cen, C Yang, J Ren; (VI) \\ Manuscript writing: All authors; (VII) Final approval of manuscript: All authors. \\ \#These authors contributed equally to this work. \\ Correspondence to: Dr. Conghua Xie. Department of Radiation and Medical Oncology, Zhongnan Hospital of Wuhan University, 169 Donghu \\ Road, Wuhan 430071, China. Email: chxie_65@whu.edu.cn; Dr. Yan Gong. Department of Biological Repositories, Zhongnan Hospital of Wuhan \\ University, 169 Donghu Road, Wuhan 430071, China. Email: yan.gong@whu.edu.cn.
}

Background: The role of additional chemotherapy in pulmonary sarcomatoid carcinoma (PSC) is
controversial. This study aimed to investigate the function of chemotherapy in PSC patients with surgical
resection.
Methods: PSC patient information between 2004 to 2016 was extracted from the Surveillance,
Epidemiology, and End Results (SEER) database. X-tile software was used to calculate the optimal cut-off
value to divide groups. The disease stages were recalculated according to the American Joint Commission on
Cancer (AJCC) $8^{\text {th }}$ edition tumor-node-metastasis (TNM) staging system. Propensity score matching (PSM)
analysis was conducted to balance the baseline of patients. Kaplan-Meier analysis and Cox proportional
hazards analysis were used to evaluate survival outcome. Results: A total of 865 PSC patients were included in our study. Among them, 611 patients were only operated with surgery, and the 254 others were treated with additional chemotherapy. The median age was 69.0 years (interquartile range, 61.6 to 76.3 years). Kaplan-Meier analysis showed that patients with additional chemotherapy had longer overall survival (OS) and cancer-specific survival (CSS, $\mathrm{P}<0.05)$. The median OS and the 1-, 3-, 5-year OS rates were 36.0 months (95\% CI: 20.5-51.5 months), 72.7\%, 49.6\% and $38.5 \%$ in the chemotherapy group and 29.0 months (95\% CI: $23.6-34.4$ months), $63.2 \%, 44.5 \%$ and $37.6 \%$ in the non-chemotherapy group, respectively. The OS advantage of chemotherapy was not statistically significant after PSM analysis. Moreover, Cox proportional hazards model showed that chemotherapy was an independent prognosis factor for better OS and CSS. In subgroup of stages II and III, the chemotherapy group had a survival advantage $(\mathrm{P}<0.05)$. Patients with young age, female gender, low histology grade, large tumor size and lobectomy surgical resection benefited more from chemotherapy.

Conclusions: Chemotherapy is recommended for stages II and III PSC patients undergoing surgery, especially for those with young age, female gender, low histology grade, large tumor size and lobectomy surgical resection.

Keywords: Chemotherapy; pulmonary sarcomatoid carcinoma (PSC); Surveillance, Epidemiology, and End Results (SEER) database; survival

\footnotetext{
^ ORCID: Yanhong Cen, 0000-0001-9444-2747; Yan Gong, 0000-0002-4805-0459; Conghua Xie, 0000-0001-6623-9864.
} 
Submitted Apr 10, 2020. Accepted for publication Sep 29, 2020.

doi: $10.21037 /$ atm-20-3226

View this article at: http://dx.doi.org/10.21037/atm-20-3226

\section{Introduction}

Pulmonary sarcomatoid carcinoma (PSC) is a rare group of non-small cell lung cancer (NSCLC) and accounts for less than $1 \%$ of lung cancer (1). Five subgroups are included in PSC according to the 2015 World Health Organization Classification of Lung Tumors: pleomorphic carcinoma, spindle cell carcinoma, giant cell carcinoma, carcinosarcoma and pulmonary blastoma (2). PSC is an aggressive tumor with a $12.6-34.6 \% 5$-year overall survival (OS) (3-5). Surgery is currently the most popular strategy, especially for PSC at early stages. However, a retrospective research revealed that the median recurrence-free survival after resection was only 6.8 months (6). Chemotherapy combined with surgical resection is a standard treatment for NSCLC patients at stages IIB, IIIA and IIIB (7). No guideline or consensus has been addressed for PSC. Due to the different pathological characteristics and behaviors, the investigation on PSC remains of great importance for individualized therapy.

The benefits of additional chemotherapy on PSC patients undergoing surgery are still controversial. Due to the rarity of PSC, all retrospective studies had limited population and long diagnostic time span (6,8-13). Currently, several open access databases with large patient population are available for cancer researchers. In this study, we investigated the advantage of additional chemotherapy in PSC patients with surgical resection by searching the Surveillance, Epidemiology, and End Results (SEER) database. Propensity score matching (PSM) analysis was used to balance the covariates distribution between treated and untreated groups. We present the following article in accordance with the STROBE reporting checklist (available at http://dx.doi.org/10.21037/atm-20-3226).

\section{Methods}

\section{Patient and data selection}

We extracted the data of patients identified as PSC from 2004 and 2016 in SEER database: incidence-SEER 18 Regs Custom Data (with additional treatment field), Nov 2018 Sub (1975-2016), using the SEER*Stat software (version 8.3.6). Positive histology was defined as PSC: pleomorphic carcinoma, 8,022/3; giant cell and spindle cell carcinoma, 8,030/3; giant cell carcinoma, 8,031/3; spindle cell carcinoma, not otherwise specified (NOS), 8032/3; pseudosarcomatous carcinoma, 8,033/3; pulmonary blastoma, 8972/3; carcinosarcoma, NOS, 8,980/3 (1). Disease primary sites and corresponding ICD-O-3 codes were main bronchus lung, C34.0; upper lobe lung, C34.1; middle lobe lung, C34.2; lower lobe lung, C34.3; over lapping lesion of lung, C34.8; lung NOS, C34.9. The exclusion criteria were as follows: (I) incomplete followup; (II) unknown survival month; (III) sources from autopsy only or death certification; (IV) received radiation therapy; (V) did not undergo surgery.

The following variates were collected: age, year at diagnosis, gender, race, grade, histological result, surgery type, tumor size, disease stage and chemotherapy status. Disease stages were adjusted manually based upon the American joint commission on cancer (AJCC) $8^{\text {th }}$ edition tumor-node-metastasis (TNM) staging system (14). The first outcome end point was OS, and the second one was cancer-specific survival (CSS). OS was calculated form the date of diagnosis to death or last follow-up. CSS was identified as the time ranging from the date of diagnosis until the date of death due to cancer.

\section{Statistical analysis}

The optimal cut-off values in age, year at diagnosis and tumor size were calculated by X-tile software (version 3.6.1) to divide patients into groups. The association between chemotherapy and clinical demographics or tumor characteristics were analyzed by chi-square tests. The survival curves were depicted by Kaplan-Meier analysis and compared by log-rank test. Univariate and multivariate analyses were performed by the Cox proportional hazards model to identify variates associated with OS and CSS, with 95\% confidence intervals (CIs). PSM analysis was conducted to balance the baseline of patients. The covariates included age, gender, race, year of diagnosis, grade, histology, surgery type, tumor size and disease stage. In subgroup analysis, Cox model was used to determine the significant difference in different characteristic and chemotherapy status. All the statistical analyses and PSM analyses were performed by 
SPSS (version 25.0) software. Forest plot was drawn up using GraphPad Prism (version 7.0). A lower than 0.05 twosided $\mathrm{P}$ value was considered as statistically significant.

\section{Results}

\section{Patient characteristics}

A total of 865 patients were included in our study before PSM (Table 1). The median age was 69.0 years (interquartile range, 61.6 to 76.3 years). All patients had a median follow-up of 36.1 months (range, 0 to 155 months). The patients receiving additional chemotherapy had younger ages, lower grade tumors, larger tumor sizes and advanced tumors. There was a significant difference in the number of patients receiving chemotherapy between different surgery type groups and year of diagnosis groups $(\mathrm{P}<0.05)$. To balance the baseline of patient features between the chemotherapy and non-chemotherapy groups, a one-totwo PSM method was conducted with a caliper of 0.02 and random matching order. After PSM, 213 patients remained in the chemotherapy group and 342 ones were matched in the non-chemotherapy group (Figure 1). The numbers of patients receiving chemotherapy in different disease stage groups showed a statistically significant difference $(\mathrm{P}<0.05)$. The other variables were all balanced after the PSM analysis $(\mathrm{P}>0.05$; Table 1).

\section{Survival analysis}

Kaplan-Meier analysis indicated that patients with additional chemotherapy had longer OS and CSS compared with the ones without chemotherapy $(\mathrm{P}<0.05$; Figure $2 A, B)$. The median OS and the 1-, 3-, 5-year OS rates were 36.0 months (95\% CI: $20.5-51.5$ months), $72.7 \%, 49.6 \%$ and $38.5 \%$ in the chemotherapy group and 29.0 months (95\% CI: $23.6-34.4$ months), $63.2 \%, 44.5 \%$ and $37.6 \%$ in the non-chemotherapy group, respectively. The median CSS and the 1-, 3-, 5-year CSS rates were 47.0 months (95\% CI: $32.2-61.9$ months), $74.7 \%, 52.3 \%$ and $41.1 \%$ in the chemotherapy group and 29.0 months (95\% CI: 24.1-33.9 months), $63.4 \%, 44.3 \%$ and $37.5 \%$ in the nonchemotherapy group, respectively. After PSM analysis, the survival advantage of chemotherapy was not significant. The OS and CSS survival curves in the chemotherapy and non-chemotherapy groups in matching cohorts are shown in Figure 2C,D.

Multivariate analysis by Cox proportional hazard model showed that chemotherapy was an independently prognostic factor for better OS. Young age, female gender, low histological grade and early disease stage were also associated with prolonged survival (Table 2). In the analysis for CSS, old age, late year of diagnosis, large tumor size, advanced disease stage and no chemotherapy were all adverse factors (Table 3).

\section{Subgroup analysis}

Considering the reduction of selection bias, all subgroup analyses were conducted using the cohorts after PSM. In the stages II and III patients, survival advantage was observed in the chemotherapy group $(\mathrm{P}<0.05)$, which was not found in the stages I and IV patients (Figure 3). The 5 -year OS rates for chemotherapy (yes $v s$. no/unknown) were $52.0 \%$ vs. $37.6 \%$ and $37.0 \%$ vs. $26.6 \%$ in the stages II and III patients. Prolonged survival time was also associated with chemotherapy in pseudosarcomatous carcinoma group. More detailed subgroup analysis in the stage IA, IB, IIA groups showed that chemotherapy benefited stage IIA PSC patients receiving surgery (Figure 4). In the analysis for CSS, chemotherapy played a positive role in patients at $65-74$ years or with large tumors $(>4 \mathrm{~cm})$. Female patients or lobectomy surgery patients might benefit more from additional chemotherapy. Patients with poor differentiation or undifferentiated histology, as well as at stages II and III or pseudosarcomatous carcinoma, were also recommended for chemotherapy. Cox proportional hazard analysis showed that chemotherapy was an independently prognostic factor of OS in patients at 65-74 years, with pseudosarcomatous carcinoma, or at stages II and III. Chemotherapy was also an independently prognostic factor of CSS in patients at $\leq 74$ years, with large tumor $(>4 \mathrm{~cm})$, at stages II and III, or with lobectomy surgery. Furthermore, additional chemotherapy independently influence the CSS of female patients or patients with poor differentiation or undifferentiated histology (Figures 5,6).

\section{Discussion}

As a subtype of NSCLC, PSC has more aggressive behaviors and worse prognosis than conventional NSCLC. The 5-year survival rate of PSC was reported to be from $20.1 \%$ to $36.7 \%(6,15-17)$. The accurate diagnosis of PSC requires histological examination of large tissues (18). Surgery is a mainstay and important treatment, especially 


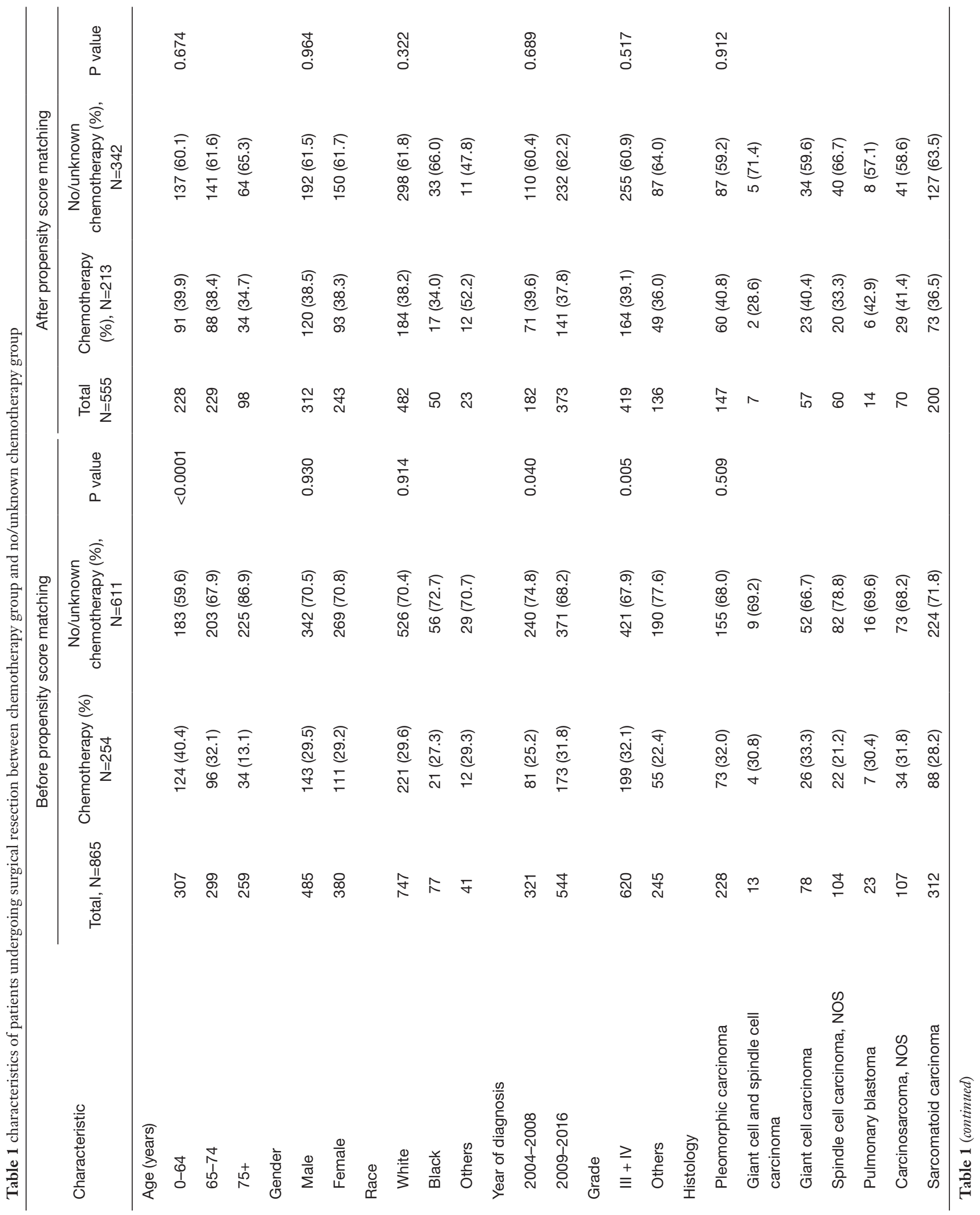




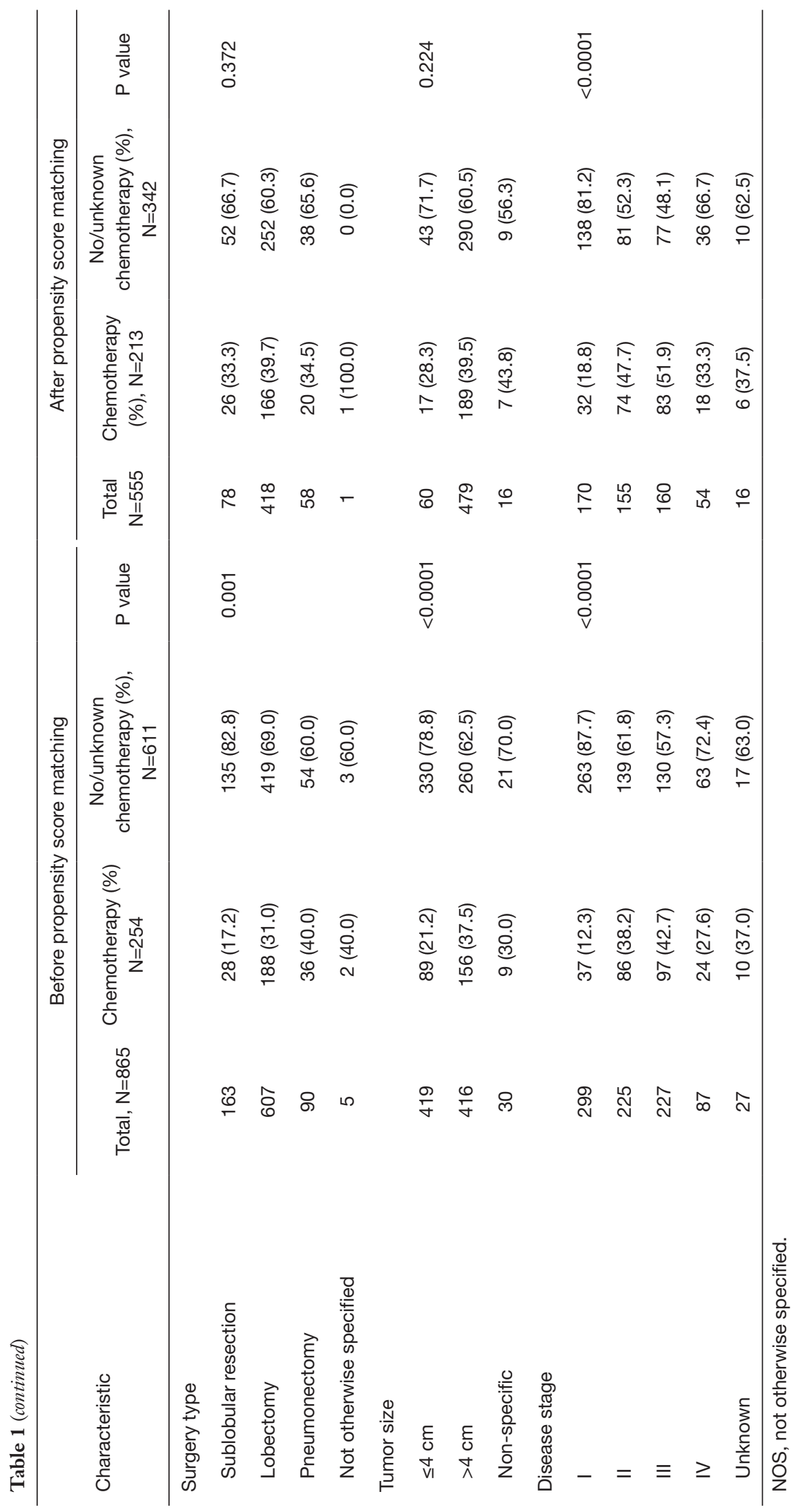




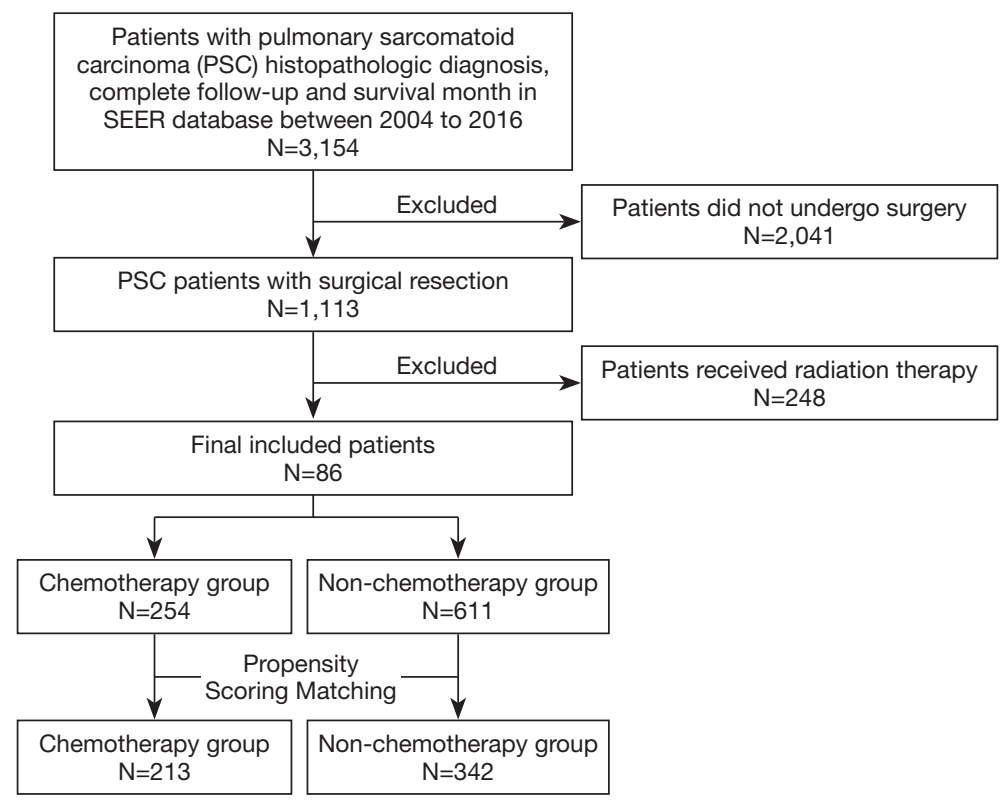

Figure 1 A flow diagram for selection of study population.
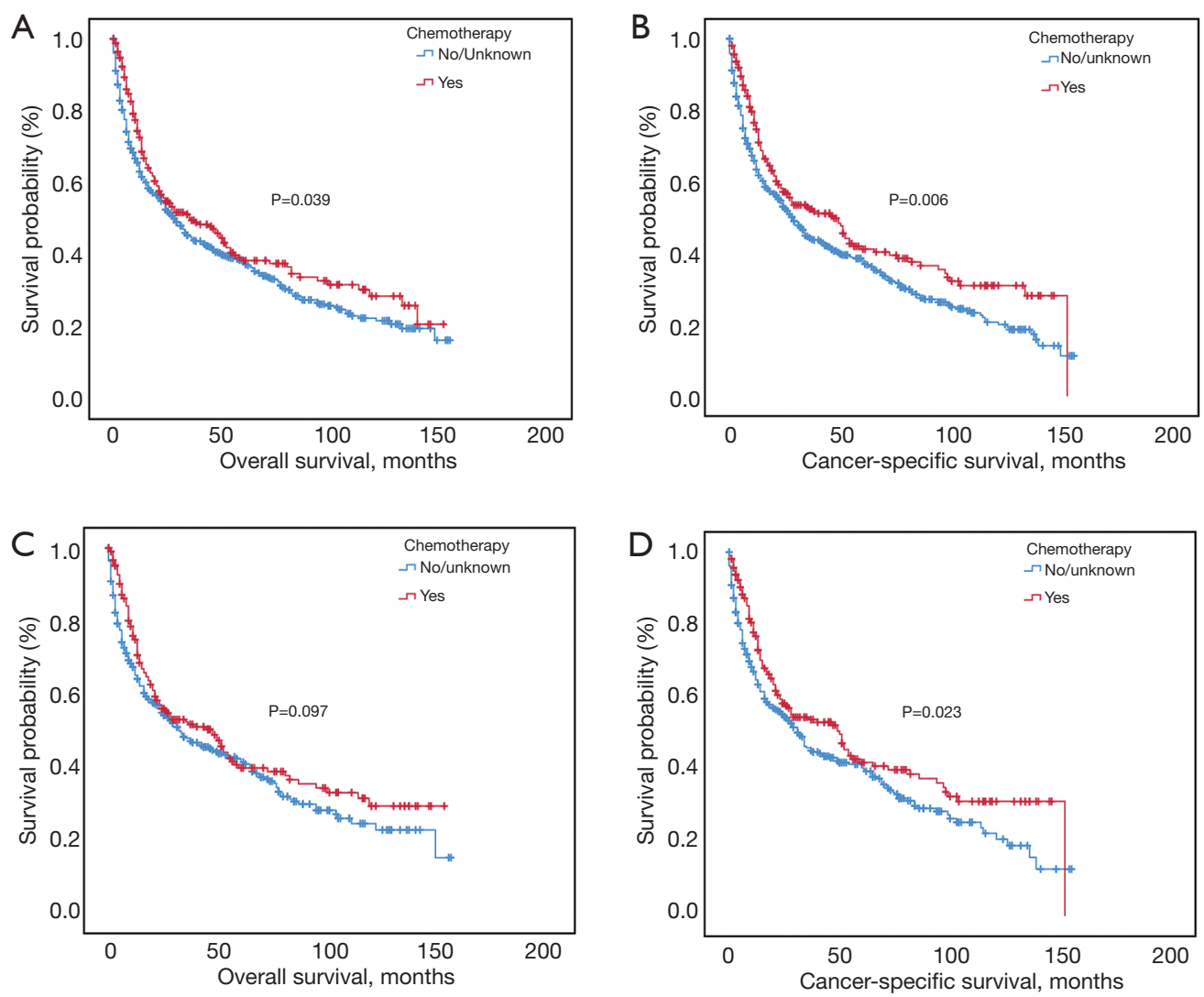

Figure 2 Overall survival and cancer-specific survival of patients according to chemotherapy treatment before (A,B) and after (C,D) PSM. PSM, propensity score matching. 
Table 2 Univariate and multivariate Cox proportional Hazard analyses for the overall survival in pulmonary sarcomatoid carcinoma patients before propensity score matching

\begin{tabular}{|c|c|c|c|c|}
\hline & \multicolumn{2}{|c|}{ Univariate analysis } & \multicolumn{2}{|c|}{ Multivariate analysis } \\
\hline $0-64$ & Reference & & Reference & \\
\hline \multicolumn{5}{|l|}{ Gender } \\
\hline Male & Reference & & Reference & \\
\hline Female & $0.779(0.656-0.925)$ & 0.004 & $0.832(0.697-0.993)$ & 0.042 \\
\hline Others & $1.007(0.668-1.518)$ & 0.973 & & \\
\hline \multicolumn{5}{|l|}{ Year of diagnosis } \\
\hline 2004-2008 & Reference & & & \\
\hline 2009-2016 & $1.112(0.913-1.329)$ & 0.242 & & \\
\hline \multicolumn{5}{|l|}{ Grade } \\
\hline III + IV & Reference & & Reference & \\
\hline Others & $0.801(0.662-0.970)$ & 0.023 & $0.789(0.648-0.961)$ & 0.019 \\
\hline Pulmonary blastoma & $0.484(0.254-0.920)$ & 0.027 & & \\
\hline Carcinosarcoma, NOS & $0.934(0.695-1.255)$ & 0.651 & & \\
\hline Sarcomatoid carcinoma & $1.157(0.929-1.440)$ & 0.193 & & \\
\hline \multicolumn{5}{|l|}{ Surgery type } \\
\hline Sublobular resection & Reference & & & \\
\hline Lobectomy & $0.692(0.561-0.854)$ & 0.001 & & \\
\hline Pneumonectomy & $0.848(0.618-1.162)$ & 0.304 & & \\
\hline \multicolumn{5}{|l|}{ Tumor size } \\
\hline$\leq 4 \mathrm{~cm}$ & Reference & & & \\
\hline$>4 \mathrm{~cm}$ & $1.403(1.179-1.668)$ & $<0.0001$ & & \\
\hline
\end{tabular}

Table 2 (continued) 
Table 2 (continued)

\begin{tabular}{|c|c|c|c|c|}
\hline & \multicolumn{2}{|c|}{ Univariate analysis } & \multicolumn{2}{|c|}{ Multivariate analysis } \\
\hline I & Reference & & Reference & \\
\hline IV & 5.279 (4.010-6.949) & $<0.0001$ & $4.766(3.524-6.445)$ & $<0.0001$ \\
\hline \multicolumn{5}{|c|}{ Chemotherapy status } \\
\hline No/unknown & Reference & & Reference & \\
\hline
\end{tabular}

NOS, not otherwise specified.

Table 3 Univariate and multivariate Cox proportional hazard analyses for cancer-specific survival in pulmonary sarcomatoid carcinoma patients before propensity score matching

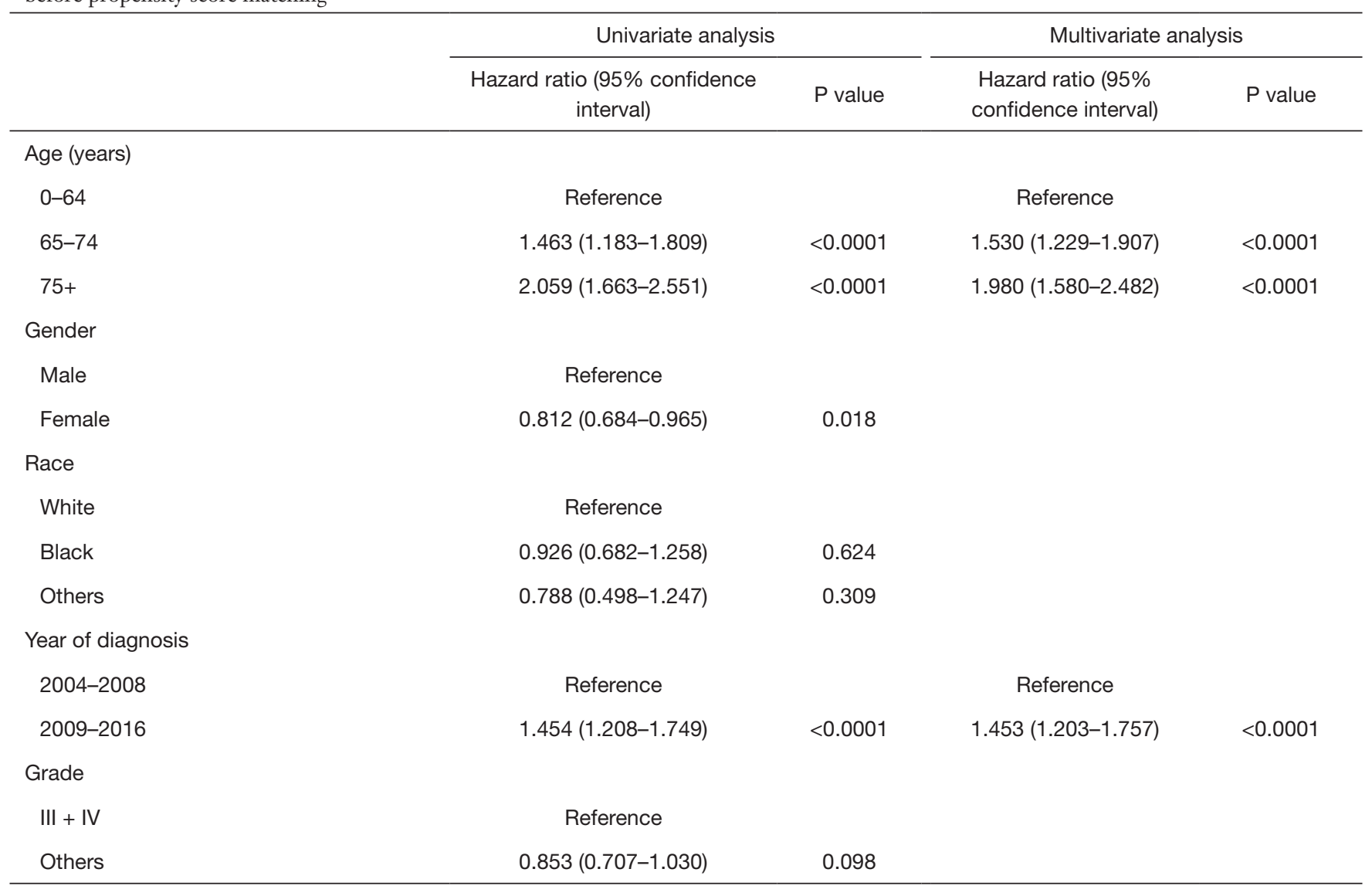

Table 3 (continued) 
Table 3 (continued)

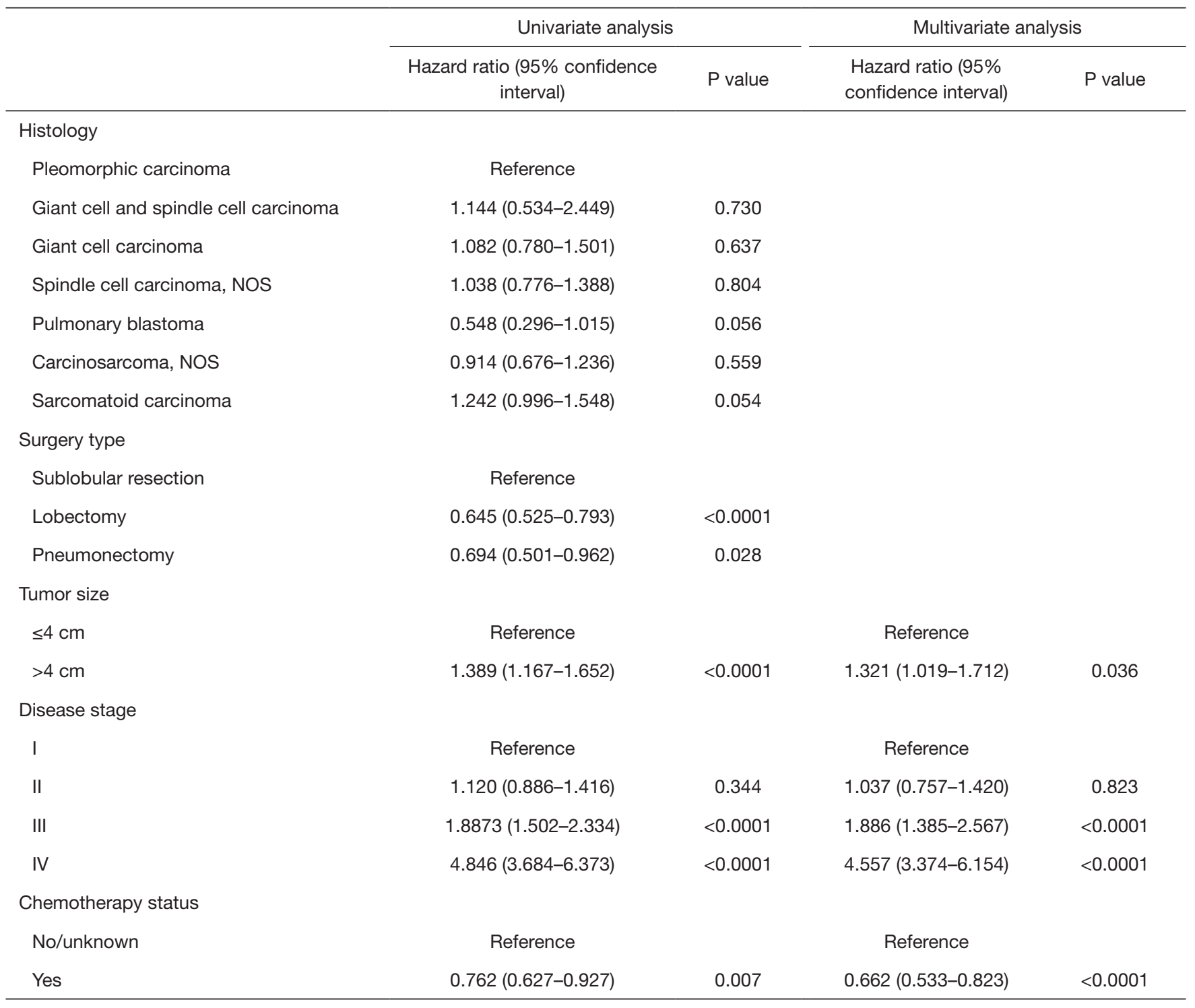

NOS, not otherwise specified.

for PSC at early stages. Surgical methods include sublobar resection, lobectomy and pneumonectomy. Sublobar resection is composed of segmentectomy and wedge resection. Compared with pneumonectomy, lobectomy has the same therapeutic effects with better preservation of lung functions for NSCLC patients. For elderly patients with early stage NSCLC, sublobar resection achieves equivalent therapeutic outcomes compared with lobectomy. Considerable research has demonstrated that surgery promotes the survival of PSC patients. Retrospective analysis of 69 PSC patients by Lin et al. found that good prognosis was associated with complete resection $(\mathrm{P}<0.05)(11)$. A more recent study reported that the median OS of PSC patients with complete surgical resection was better than that of PSC patients without surgery [16.4 months (95\% CI: 6.1 to not reached] vs. 3 months [95\% CI: 2.1-5 months)] (6). However, PSC had a high recurrence (up to $70 \%$ ) after operative treatment, and 
A
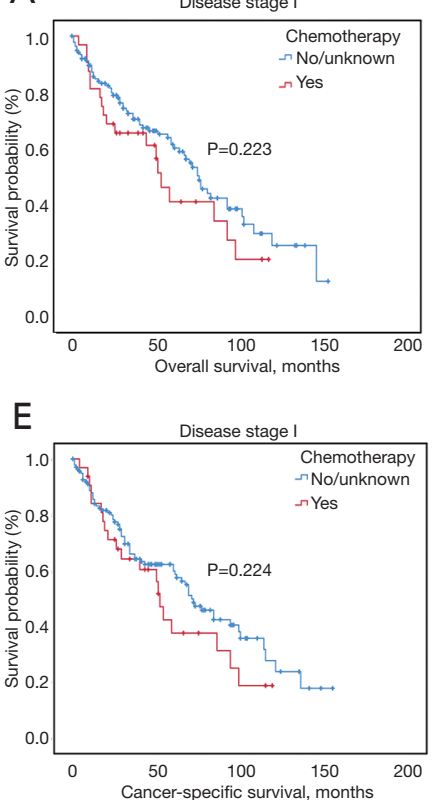

B

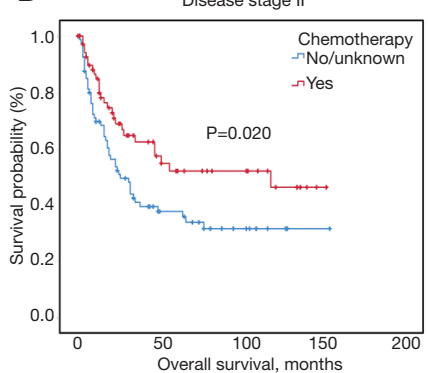

$\mathrm{F}$

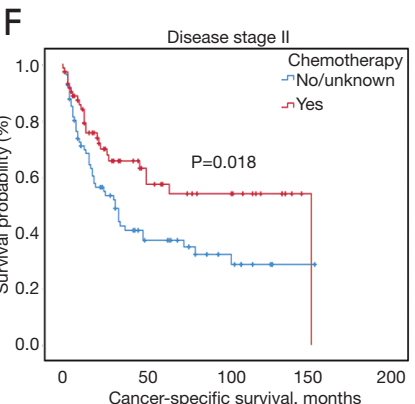

C

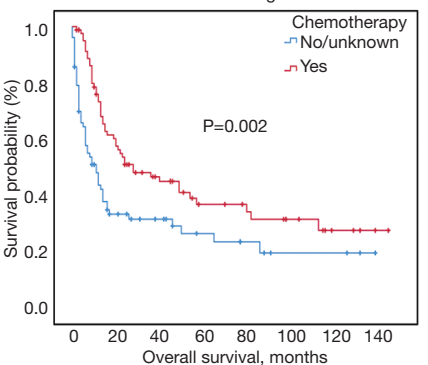

G

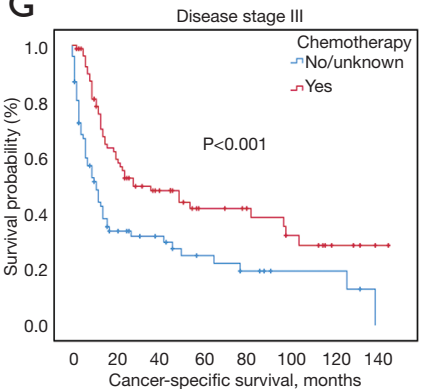

D

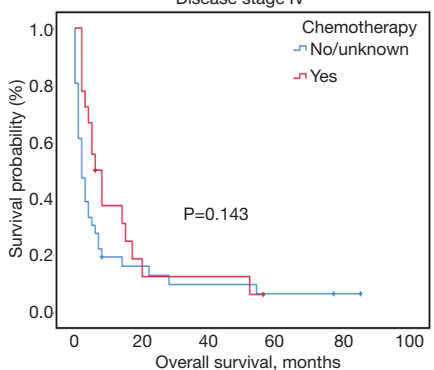

$\mathrm{H}$

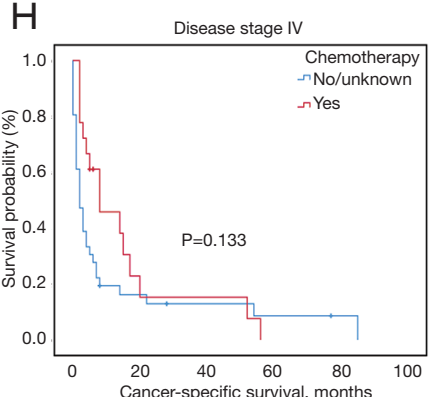

Figure 3 Univariate Kaplan-Meier analysis on overall survival and cancer-specific survival of PSC patients atin different stages:. Stage I (A,E); Stage II (B,F); Stage III (C,G); Stage IV (D,H). PSC, pulmonary sarcomatoid carcinoma.

A
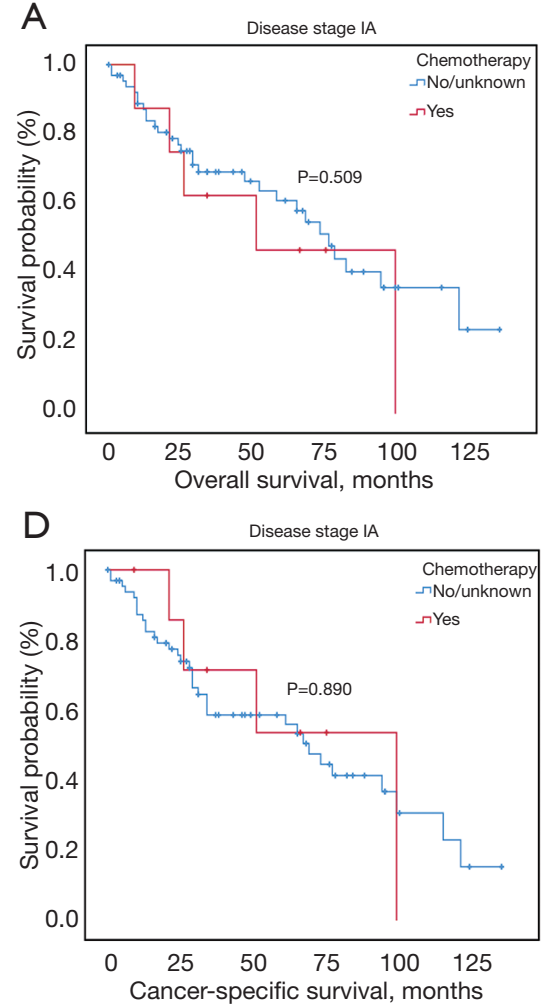

B

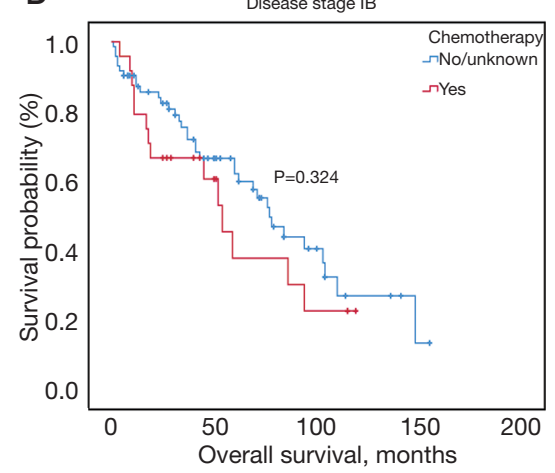

E

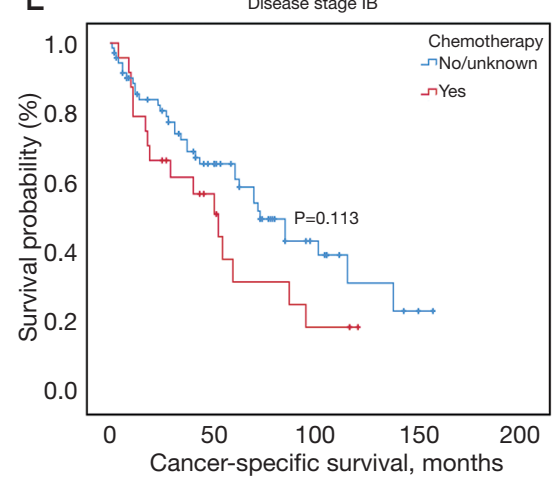

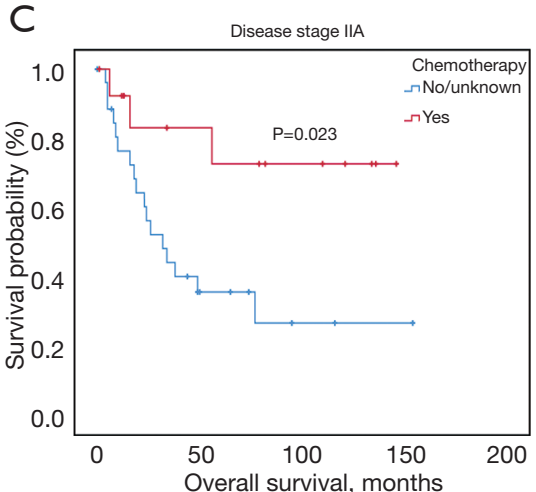

$\mathrm{F}$

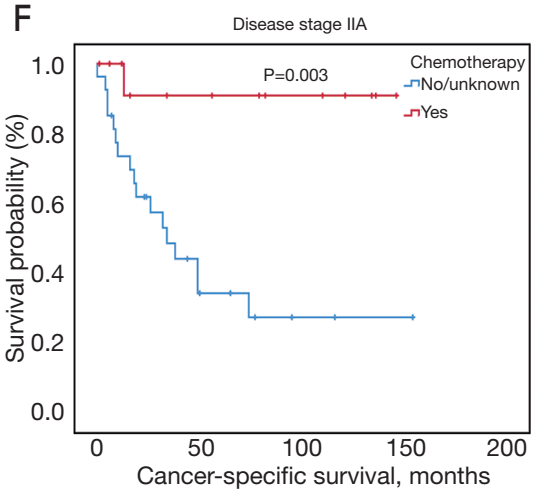

Figure 4 Subgroup Kaplan-Meier analysis on overall survival and cancer-specific survival of PSC patients inat different stages:. Stage IA (A,D); Stage IB (B,E); Stage IIA (C,F). PSC, pulmonary sarcomatoid carcinoma. 


\section{Subgroup analysis for OS}
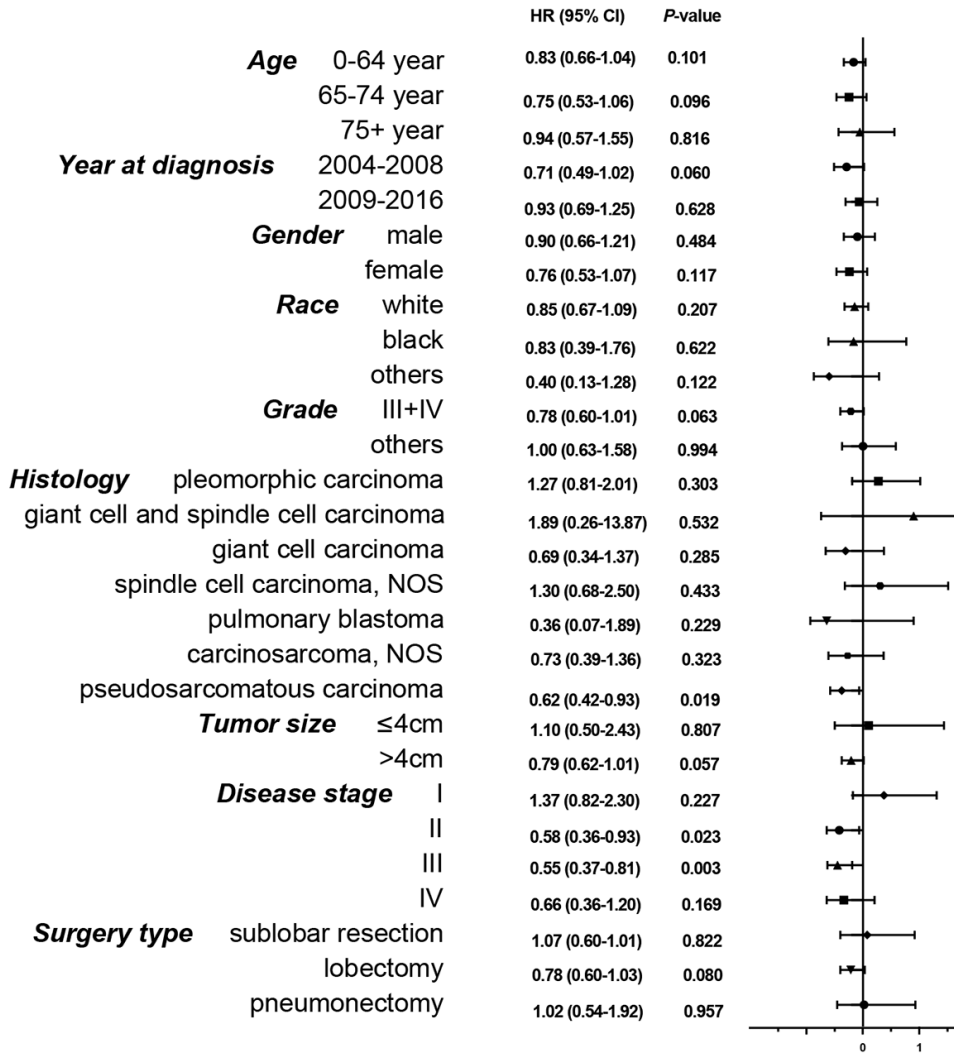

Chemtherapy Better

No/Unknown Chemtherapy Better

Figure 5 Subgroup Cox proportional hazard analysis on overall survival of PSC patients according to chemotherapy status. PSC, pulmonary sarcomatoid carcinoma; NOS, not otherwise specified.

distant metastasis was found more frequently than local metastasis $(3,6,19)$. To improve PSC prognosis, combined therapy should be considered. In consideration of recurrence and metastasis, we conjectured that perioperative chemotherapy would have a better performance in efficacy than radiotherapy. However, it was still controversial whether additional chemotherapy was an independent factor for good prognosis in PSC patients or not (Table 4) $(3,6,8,9,11-13,20)$. Our studies suggested the therapeutic values of perioperative chemotherapy for PSC patients.

In our study, most patients were male, in their 60 s and classified as pleomorphic carcinoma. A total of 254 patients received chemotherapy, most of whom were younger, earlier diagnosed, with lower histology grade, larger tumor size, advance stage and had received lobectomy. Surprisingly, only $40 \%$ patients at stages II and III received chemotherapy, which might be associated with negative results of previous studies. We examined the temporal trends, excluding an overrepresentation from an earlier era. Our results suggested that chemotherapy improved the survival of PSC patients with surgery, supporting the results of previous small sample studies. We used both Cox proportional hazard model and PSM model to compare the OS and CSS between chemotherapy and no/unknown chemotherapy patients. In the PSM model, perioperative chemotherapy only showed an OS advantage with no statistical significance, perhaps due to the other uncontrolled confounding factors or limited sample sizes. To explore the proper patients, subgroup analysis was performed further. Our data suggested that patients at stage II and III with perioperative chemotherapy had a significant improvement in the OS and CSS. In a more detailed subgroup analysis, we observed a survival benefit in patients at stage IIA, but not at earlier stages (IA and IB). 


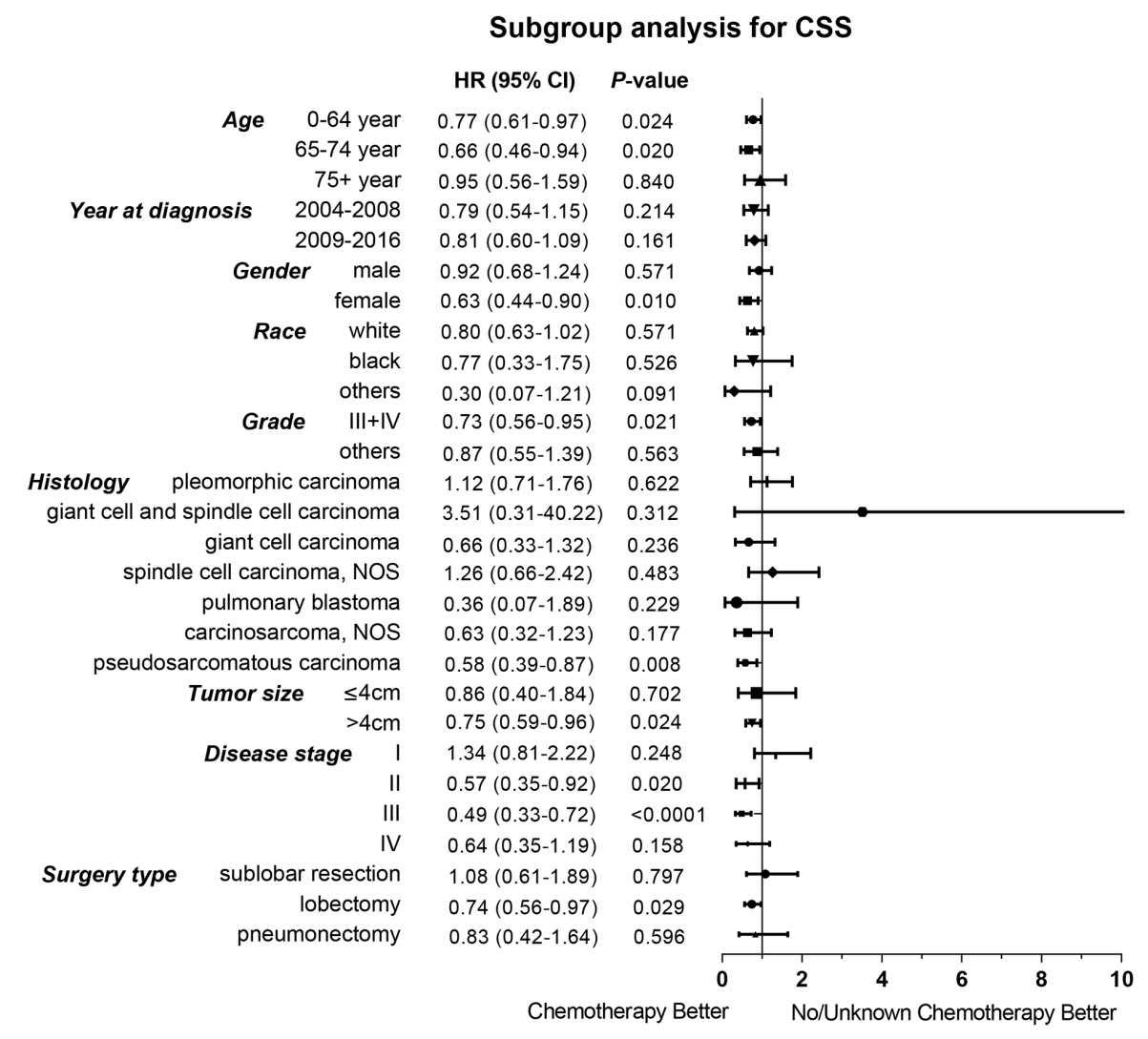

Figure 6 Subgroup Cox proportional hazard analysis on cancer-specific survival of PSC patients according to chemotherapy status. PSC, pulmonary sarcomatoid carcinoma; NOS, not otherwise specified.

It is interesting that patients at stage I, receiving sublobar resection and histological classified as "pleomorphic carcinoma", "giant cell and spindle cell carcinoma" and "spindle cell carcinoma, NOS" tend to have a worse prognosis after additional chemotherapy. The National Comprehensive Cancer Network (NCCN) clinical practice guideline in NSCLC recommended chemotherapy for NSCLC patients undergoing surgical resection and with stages IB, II and III diseases. Compared to conventional NSCLC, PSC seemed to be moderately sensitive to chemotherapy.

Our finding is contradictory with some of the literature. A published study by Karim et al. showed that patients with surgery alone had the best median OS of 713.5 days, longer than 457.6 days of the ones with additional chemotherapy (12). Another study reported that 99 patients received chemotherapy after surgery, but no obvious survival advantage was provided by adjuvant chemotherapy (3).
The response to perioperative chemotherapy was poor in the Ung et al. study (6). All these previous studies were retrospective. Perioperative chemotherapy performed in patients who had higher clinical stages or other confounders were not balanced. Our research used the PSM model to balance baseline characteristics, and subgroup analysis were performed to control confounding factors.

Our study is one of retrospective studies with the largest patient population, and the first to use a PSM cohort to minimize confounding factors in PSC. However, we were aware of several limitations. First, as a retrospective study, it was unavoidable to have some biases on patient selection, although we used PSM to minimize confounding Second, the SEER database lacked general condition/performance status for true matching. Finally, our analysis was only based on the available data in one database. The number of cases in subgroup analysis was limited. Further clinical prospective trails are needed to confirm the findings of our 


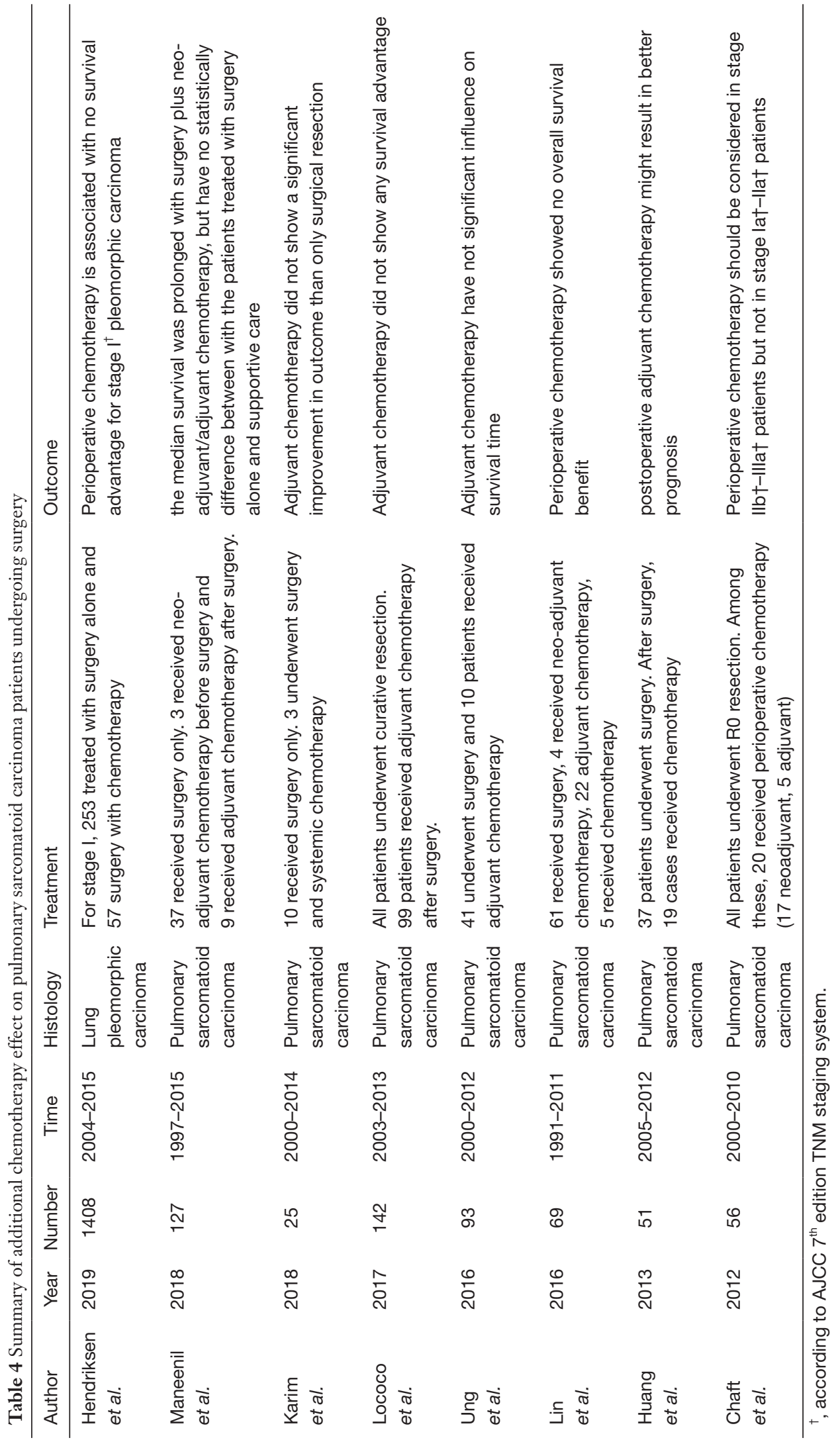


study.

\section{Conclusions}

Chemotherapy benefits stages II and III PSC patients undergoing surgical resection. Additional chemotherapy should be considered for PSC patients at stages II and III, especially for the patients with young age, female gender, poor differentiation or undifferentiated histology, large tumor size and lobectomy surgical resection.

\section{Acknowledgments}

Funding: This work was supported by National Natural Science Foundation of China (grant numbers 81773236, 81800429, 81972852), Key Research \& Development Project of Hubei Province (grant number 2020BCA069), Nature Science Foundation of Hubei Province (grant number 2020CFB612), Health Commission of Hubei Province Medical Leading Talent Project, Health Commission of Hubei Province Scientific Research Project (grant numbers WJ2019H002, WJ2019Q047), Young \& Middle-Aged Medical Backbone Talents of Wuhan (WHQG201902), and Zhongnan Hospital of Wuhan University Science, Technology and Innovation Seed Fund (grant numbers znpy2018028, znpy2018070, znpy2019001, znpy2019048 and ZNJC201922).

\section{Footnote}

Reporting Checklist: The authors have completed the STROBE reporting checklist. Available at http://dx.doi. org/10.21037/atm-20-3226

Peer Review File: Available at http://dx.doi.org/10.21037/ atm-20-3226

Conflicts of Interest: All authors have completed the ICMJE uniform disclosure form (available at http://dx.doi. org/10.21037/atm-20-3226). The authors have no conflicts of interest to declare.

Ethical Statement: The authors are accountable for all aspects of the work in ensuring that questions related to the accuracy or integrity of any part of the work are appropriately investigated and resolved. Since any information in the SEER database was anonymized data, no ethical approval requirements were needed in our study. The study was conducted in accordance with the
Declaration of Helsinki (as revised in 2013).

Open Access Statement: This is an Open Access article distributed in accordance with the Creative Commons Attribution-NonCommercial-NoDerivs 4.0 International License (CC BY-NC-ND 4.0), which permits the noncommercial replication and distribution of the article with the strict proviso that no changes or edits are made and the original work is properly cited (including links to both the formal publication through the relevant DOI and the license). See: https://creativecommons.org/licenses/by-nc-nd/4.0/.

\section{References}

1. Travis WD, Brambilla E, Nicholson AG, et al. The 2015 World Health Organization Classification of Lung Tumors: Impact of Genetic, Clinical and Radiologic Advances Since the 2004 Classification. J Thorac Oncol 2015;10:1243-60.

2. Beasley MB, Brambilla E, Travis WD. The 2004 World Health Organization classification of lung tumors. Semin Roentgenol 2005;40:90-7.

3. Lococo F, Rapicetta C, Cardillo G, et al. Pathologic Findings and Long-Term Results After Surgical Treatment for Pulmonary Sarcomatoid Tumors: A Multicenter Analysis. Ann Thorac Surg 2017;103:1142-50.

4. Weissferdt A, Kalhor N, Correa AM, et al. "Sarcomatoid" carcinomas of the lung: a clinicopathological study of 86 cases with a new perspective on tumor classification. Hum Pathol 2017;63:14-26.

5. Yin J, Yang Y, Ma K, et al. Clinicopathological characteristics and prognosis of pulmonary pleomorphic carcinoma: a population-based retrospective study using SEER data. J Thorac Dis 2018;10:4262-73.

6. Ung M, Rouquette I, Filleron T, et al. Characteristics and Clinical Outcomes of Sarcomatoid Carcinoma of the Lung. Clin Lung Cancer 2016;17:391-7.

7. NCCN.org. National Comprehensive Cancer Network (NCCN) Clinical practice guidelines in oncology. NonSmall Cell Lung Cancer. Version 3. 2020; c2020. Available online: https://www.nccn.org/store/login/login. aspx?ReturnURL=https://www.nccn.org/professionals/ physician_gls/pdf/nscl.pdf

8. Huang SY, Shen SJ, Li XY. Pulmonary sarcomatoid carcinoma: a clinicopathologic study and prognostic analysis of 51 cases. World J Surg Oncol 2013;11:252.

9. Maneenil K, Xue Z, Liu M, et al. Sarcomatoid Carcinoma of the Lung: The Mayo Clinic Experience in 127 Patients. Clin Lung Cancer 2018;19:e323-33. 
10. Gu L, Xu Y, Chen Z, et al. Clinical analysis of 95 cases of pulmonary sarcomatoid carcinoma. Biomed Pharmacother 2015;76:134-40.

11. Lin Y, Yang H, Cai Q, et al. Characteristics and Prognostic Analysis of 69 Patients With Pulmonary Sarcomatoid Carcinoma. Am J Clin Oncol 2016;39:215-22.

12. Karim NA, Schuster J, Eldessouki I, et al. Pulmonary sarcomatoid carcinoma: University of Cincinnati experience. Oncotarget 2018;9:4102-8.

13. Hendriksen BS, Hollenbeak CS, Reed MF, et al. Perioperative chemotherapy is not associated with improved survival in stage I pleomorphic lung cancer. J Thorac Cardiovasc Surg 2019;158:581-91.e11.

14. Goldstraw P, Chansky K, Crowley J, et al. The IASLC Lung Cancer Staging Project: Proposals for Revision of the TNM Stage Groupings in the Forthcoming (Eighth) Edition of the TNM Classification for Lung Cancer. J Thorac Oncol 2016;11:39-51.

Cite this article as: Cen Y, Yang C, Ren J, Gong Y, Xie C. Additional chemotherapy improves survival in stage II-III pulmonary sarcomatoid carcinoma patients undergoing surgery: a propensity scoring matching analysis. Ann Transl Med 2021;9(1):24. doi: 10.21037/atm-20-3226
15. Yendamuri S, Caty L, Pine M, et al. Outcomes of sarcomatoid carcinoma of the lung: a Surveillance, Epidemiology, and End Results Database analysis. Surgery 2012;152:397-402.

16. Martin LW, Correa AM, Ordonez NG, et al. Sarcomatoid carcinoma of the lung: a predictor of poor prognosis. Ann Thorac Surg 2007;84:973-80.

17. Weissferdt A. Pulmonary Sarcomatoid Carcinomas: A Review. Adv Anat Pathol 2018;25:304-13.

18. Baldovini C, Rossi G, Ciarrocchi A. Approaches to Tumor Classification in Pulmonary Sarcomatoid Carcinoma. Lung Cancer (Auckl) 2019;10:131-49.

19. Park JS, Lee Y, Han J, et al. Clinicopathologic outcomes of curative resection for sarcomatoid carcinoma of the lung. Oncology 2011;81:206-13.

20. Chaft JE, Sima CS, Ginsberg MS, et al. Clinical outcomes with perioperative chemotherapy in sarcomatoid carcinomas of the lung. J Thorac Oncol 2012;7:1400-5. 